SUBJECT AREAS:

MAGNETIC PROPERTIES

AND MATERIALS

FERROMAGNETISM

Received

21 March 2014

Accepted

30 May 2014

Published

18 June 2014

Correspondence and requests for materials should be addressed to

J.P.L. (pliu@uta.edu)

\section{High Energy Product Developed from Cobalt Nanowires}

\author{
Kinjal Gandha, Kevin Elkins, Narayan Poudyal, Xubo Liu \& J. Ping Liu
}

Department of Physics, University of Texas at Arlington, Arlington, TX 76019, USA.

Cobalt nanowires with high aspect ratio have been synthesized via a solvothermal chemical process. Based on the shape anisotropy and orientation of the nanowire assemblies, a record high room-temperature coercivity of $10.6 \mathrm{kOe}$ has been measured in Co nanowires with a diameter of about $15 \mathrm{~nm}$ and a mean length of $200 \mathrm{~nm}$. As a result, energy product of the wires reaches 44 MGOe. It is discovered that the morphology uniformity of the nanowires is the key to achieving the high coercivity and high energy density. Nanowires of this type are ideal building blocks for future bonded, consolidated and thin film magnets with high energy density and high thermal stability.

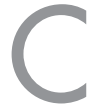

onventional wisdom on permanent magnets development is based on the concept that high magnetocrystalline anisotropy (derived from an unsymmetrical crystalline structure) of a ferromagnetic material is essential for developing high magnetic coercivity that is required for high energy product - the figure of merit of a permanent magnet. For this reason, current permanent magnets having high energy products are all based on rare-earth compounds that have extraordinarily high magneto-crystalline anisotropy ${ }^{1}$. The rare-earth compounds, however, have also some disadvantages as permanent magnets, for instance the relatively low magnetization and limited thermal stability compared to ferromagnetic $3 \mathrm{~d}$ transition metals like Fe, Co and their alloys. Recently, there is also increasing concern over future rare-earth supplies with the ever expanding demand on high performance permanent magnets that have found large new application markets in renewable energy and clean energy ${ }^{2}$. Nevertheless, the $3 \mathrm{~d}$ elements and their alloys, though cheaper and more abundant compared to rare-earth elements, are known as "soft magnetic materials" because of their high saturation magnetization, high Curie temperatures and low coercivity (derived from their low magnetocrytalline anisotropy).

It has been described like a dream to develop high coercivity in $3 \mathrm{~d}$ transition metals and their alloys for use as permanent magnets. If this goal can be made, all the disadvantages and problems related to rare-earth magnets will be resolved. Theoretical calculations showed that giant magnetic anisotropy is possible in tetragonal $\mathrm{FeCo}$ alloys $^{3}$, though experimental evidence needs to be further sought. On another front, making use of shape anisotropy of ferromagnets to develop coercivity has been proved to be feasible. The best example may be the Alnico permanent magnets that have been produced since $1930 \mathrm{~s}^{4}$. In an Alnico magnet, the microstructure is primarily composed of two nanoscale phases formed through Spinodal decomposition: isolated needles of ferromagnetic FeCo-rich phase and a non-magnetic matrix of NiAl-rich phase. However, performance of Alnico magnets is still restricted by their very modest coercivity (typical magnetic properties of commercial Alnico magnets have their coercivity Hci $<1.5$ kOe and energy product $(\mathrm{BH})_{\mathrm{m}}<10$ MGOe).

Extensive research in recent years in magnetic nanoparticles especially in magnetic nanowires and nanorods ${ }^{5-9}$ has renewed the interests in developing high coercivity in transition metal nanocrystals based on shape anisotropy. Electrochemical deposition ${ }^{10-12}$ and chemical synthesis ${ }^{13-17}$ are widely adopted to produce Co and Fe based ferromagnetic nanowires and nanorods with enhanced coercivity. Room-temperature coercivities up to $7.0 \mathrm{kOe}$ have been reported for aligned single-crystalline Co nanorods ${ }^{17}$.

In this letter, we report high coercivity exceeding $10 \mathrm{kOe}$ at room temperature developed from Co nanowires prepared via a bottom-up solvothermal process.

\section{Results}

Figure 1 shows the morphology of the Co nanowires synthesized via a solvothermal chemical process. X-ray diffraction shows that the wires have an expected hcp crystal structure. As shown in Fig. 1a and 1b, the Co nanowires have cylinder shapes with ellipsoidal tips. The wires have typical size about 200 to $300 \mathrm{~nm}$ in length and an average diameter of $15 \mathrm{~nm}$ from the TEM observation. The high resolution TEM results (Fig. 1c) indicate 

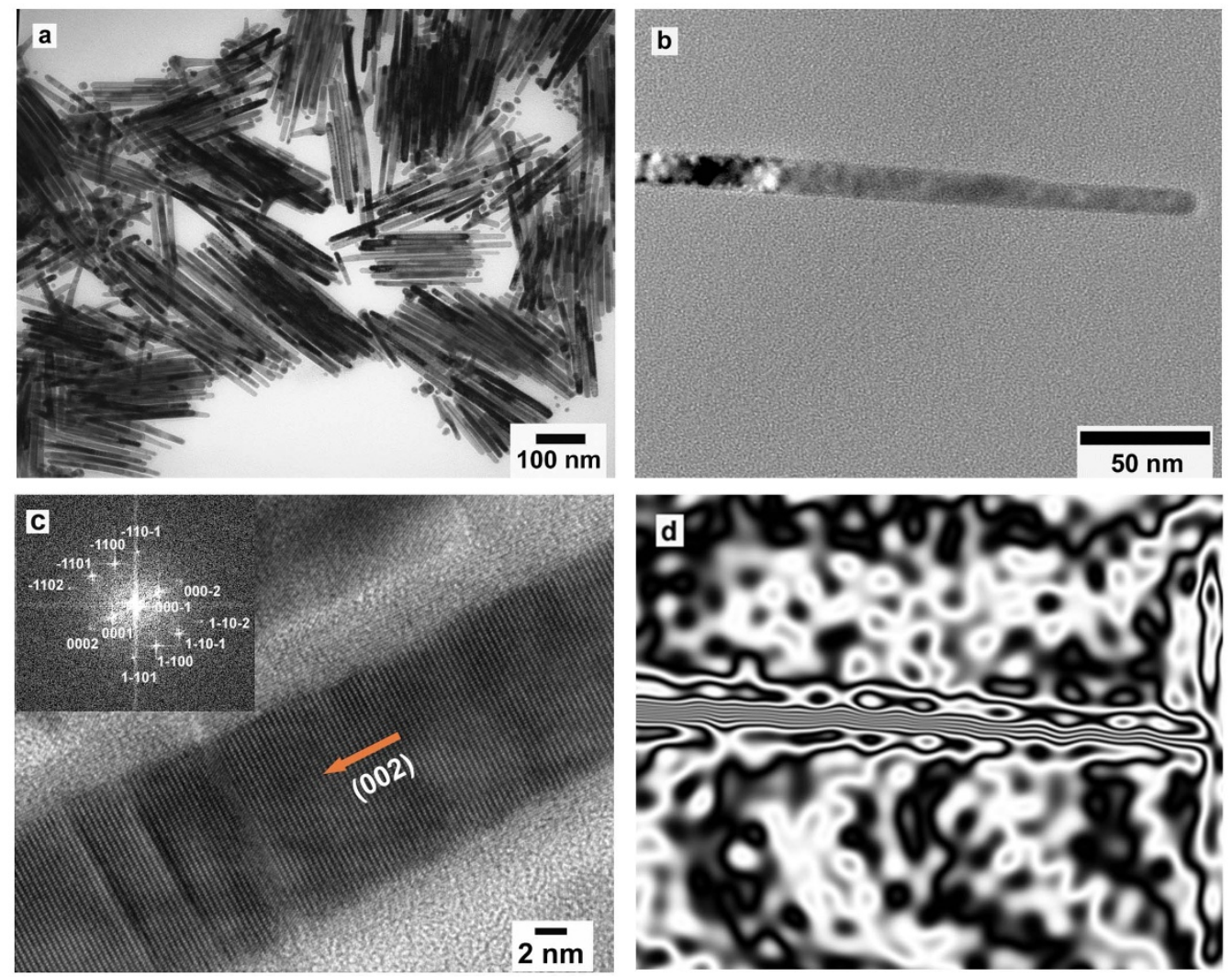

Figure 1 TEM analysis of Cobalt nanowires. (a), Bright-field TEM image of the Co nanowires. (b), Bright-field TEM image of an end-tip single Co nanowire. (c), High resolution TEM image of a single Co nanowire in [11-20] zone axis. Inset the corresponding numerical FFT pattern. (d), Hologram of a single Co nanowire (with the same magnification as (b)).

that each wire is a single crystal with the c-axis (002), or the easy magnetization direction, along the long axis of the wire. The magnetic holography image (Fig. 1d) reveals a single-domain structure and confirms the magnetization orientation along the long axis. This magnetocrystalline configuration, coupled with the shape of high aspect ratio, favors a coherent rotation of the magnetization process and thus should lead to large coercivity of the nanowire assemblies.

In the case of an ellipsoid ferromagnet, if one assumes a coherent rotation of the magnetization, the shape anisotropy field $\mathrm{H}_{\mathrm{A}}$ is given by $^{18}$

$$
\mathrm{H}_{\mathrm{A}}=\mathrm{J}_{\mathrm{s}}\left(\mathrm{N}_{\mathrm{a}}-\mathrm{N}_{\mathrm{c}}\right)
$$

where $\mathrm{N}_{\mathrm{a}}$ and $\mathrm{N}_{\mathrm{c}}$ are the de-magnetizing factors along the short and long axes, respectively. $\mathrm{J}_{\mathrm{s}}$ is the magnetic induction and it is $18 \mathrm{kG}$ for Co at room temperature. For infinitely long ellipsoids $\mathrm{N}_{\mathrm{a}}=1 / 2$ and $\mathrm{N}_{\mathrm{c}}=0$. If the long-axis direction of a Co nanowire conforms to its magnetocrystalline uniaxial direction [0001] (c-axis, or normal direction of the (002) planes), as observed in Fig. 1, the maximum magnetic anisotropy field contributed from the shape anisotropy will be $9 \mathrm{kOe}$. Plus the anisotropy field of $7.6 \mathrm{kOe}$ contributed by the magnetocrystalline anisotropy of bulk cobalt ${ }^{19}$, a total anisotropy field up to $16.6 \mathrm{kOe}$ at room temperature may be reached, which shows the upper limit of coercivity that can be developed from Co nanocrystals with high aspect ratio.

Figure 2a shows the magnetic hysteresis loop at room temperature of a randomly oriented Co nanowire assembly. X-ray diffraction of the assembly is shown beneath in Fig. 2c. Significant coercivity exceeding $6.5 \mathrm{kOe}$ is observed, attributed to the magnetocrystalline anisotropy and the shape anisotropy. Although bulk Co material has an anisotropy field of $7.6 \mathrm{kOe}$, it is practically difficult to obtain a coercivity value of $6.5 \mathrm{kOe}$ for any bulk cobalt materials. Shape anisotropy has played a substantial role in magnetic hardening of the nanowire assembly.

Another character of the magnetization loop shown in Fig. 2a is the remanent magnetization ratio, $\mathrm{M}_{\mathrm{r}} / \mathrm{M}_{\mathrm{s}}$ (ratio of the remanent magnetization over the saturation magnetization). According to the Stoner-Wohlfarth model $^{20}$, in a randomly oriented singledomain particle system, the $\mathrm{M}_{\mathrm{r}} / \mathrm{M}_{\mathrm{s}}$ ratio is 0.5 , which is exactly the situation observed in Fig. 2a. A corresponding parameter that also reflects the degree of the orientation (alignment) of the domains is the "squareness" of the loop in the second quadrant, measured by the ratio of the area covered by the loop curve (demagnetization curve) and the area of the rectangular $\mathrm{M}_{\mathrm{r}} \mathrm{H}_{\mathrm{c}}$. As expected, the loop of the randomly oriented system has a modest "squareness" (0.60).

A dramatic change was observed in the hysteresis loop when the nanowires were aligned in a magnetic field (Fig. 2b). Figure $2 \mathrm{~d}$ is the $\mathrm{X}$-ray diffraction (XRD) pattern of the aligned wires along the magnetic field direction. One can see clearly from the XRD pattern that all the wires were aligned with their c-axis parallel to the magnetic field direction, while other diffraction peaks all disappear. As shown in Fig. 2b, the room-temperature coercivity of the magnetically aligned sample has increased to about $10.3 \mathrm{kOe}$, a record high coercivity value for cobalt material at room temperature, which is nearly double that of the randomly oriented sample (Fig. 2a). The remanent magnetization of the aligned sample also increased greatly, leading to the remanent magnetization ratio $\mathrm{M}_{\mathrm{r}} / \mathrm{M}_{\mathrm{s}}$ of 0.92 , which is an almost doubled value compared to the randomly aligned system (while the saturation magnetization remained unchanged). Consequently, the "squareness" of the loop is significantly increased (to 0.78). This change is a typical Stoner-Wohlfarth behavior of a single-domain particle system upon magnetic alignment ${ }^{20}$. Based on the model, for an ideal single-domain particle system with identical magnetic 
a

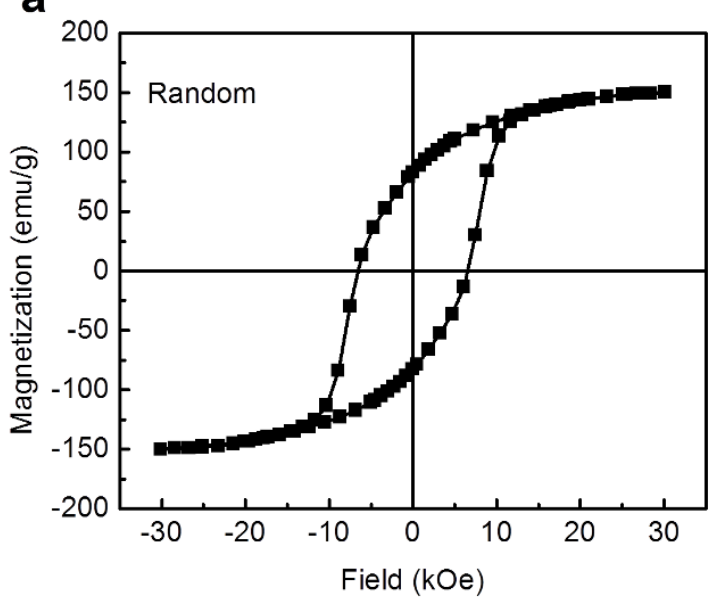

C

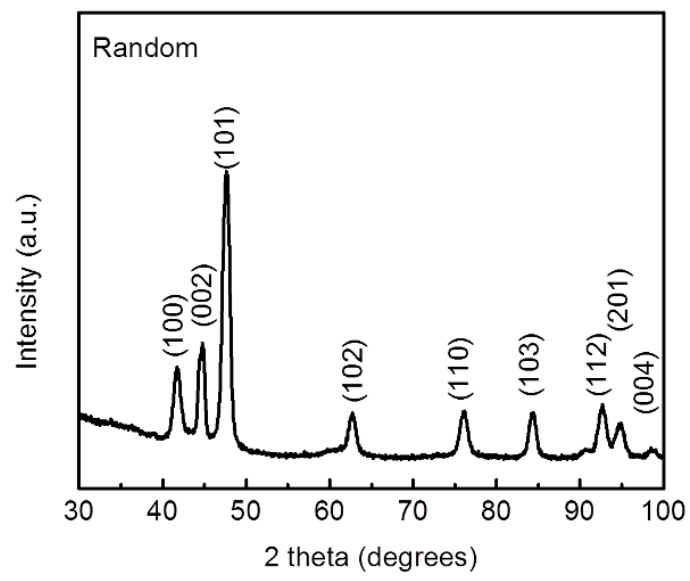

b

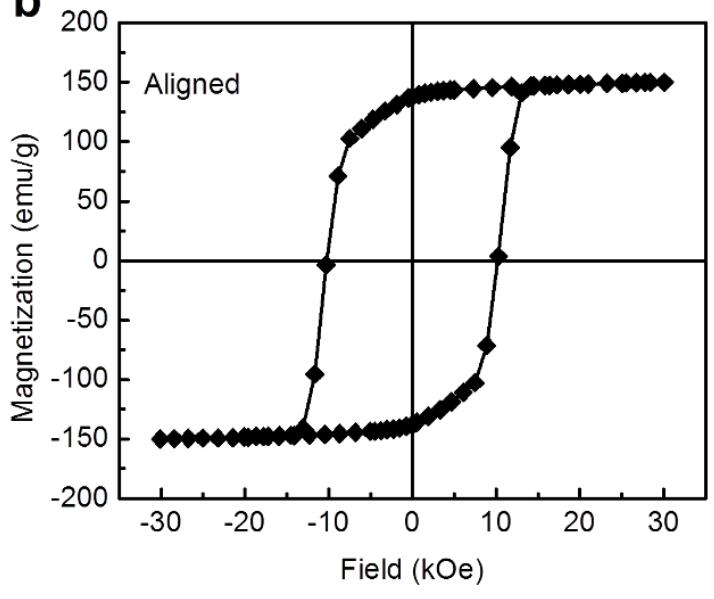

d

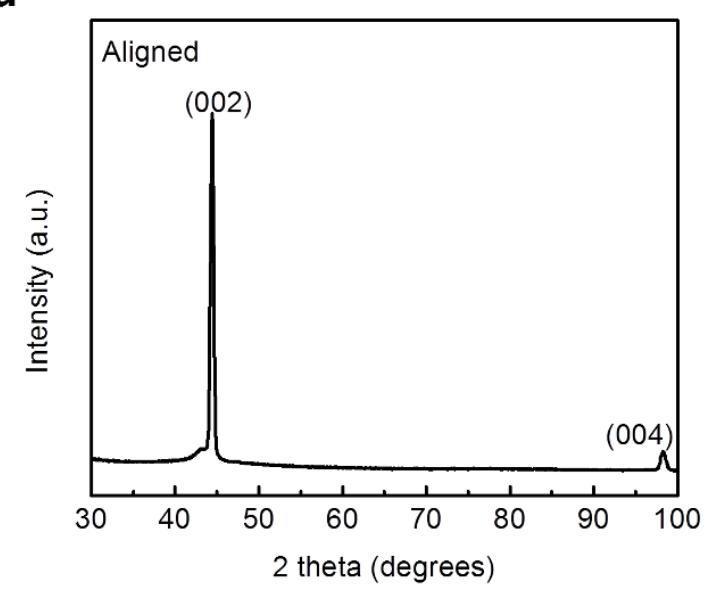

Figure $2 \mid$ Magnetic and structural characterizations. (a), Magnetization loop of a randomly oriented Co nanowire assembly at $300 \mathrm{~K}$. (b), Magnetization loop of the aligned Co nanowire assembly (along parallel direction) at $300 \mathrm{~K}$. (c), XRD pattern of the Co nanowires in random orientation. (d), XRD pattern of aligned Co nanowires in the alignment direction.

properties of the particles, the magnetically aligned assemblies will gain coercivity and remanence values twice that of the corresponding systems that are randomly oriented. This "sharp" behavior has been rarely observed in actual hard magnetic materials for the reason that identical single-domain particles are difficult to prepare experimentally. The present results also support the statement that total anisotropy field of the nanowire system is the sum of magnetocrytalline anisotropy field and shape anisotropy field (16.6 kOe in total, at room temperature). From theoretical point of view, the Co nanowire assemblies studied here deserve further systematic investigations.

From an application point of view, the Co nanowire system may be even more intriguing as a potential rare-earth-free high-temperature permanent magnet based on its extremely high Curie temperature and the stability of the Co nanorod morphology up to elevated temperatures revealed by a recent study ${ }^{21}$. In Fig. 3 the energy product of the aligned nanowires in epoxy is estimated based on a 100 percent volume fraction of closely packed Co nanowires using the theoretical density of $8.92 \mathrm{~g} / \mathrm{cm}^{3}$ containing closely packed nanowires and have obtained a $(\mathrm{BH})_{\max }$ value up to $44 \mathrm{MGOe}$. Although this result does not reflect any effects that can potentially reduce the energy density of a bulk magnet such as packing factor, demagnetization or dipolar interactions ${ }^{22}$, the Co nanowires of this type can potentially be ideal building blocks for high performance bonded magnets, consolidated magnets as well as thin film magnets with both isotropic and anisotropic magnetic structures.

To further understand the effect of morphology on the coercivity and magnetization hysteresis in the nanowire systems, we compared two groups of samples, one with more uniform morphology and one with less uniform morphology. Figure 3 shows the details of the two representative samples in magnetization and morphology. Histogram analysis (Fig. $3 \mathrm{~g}$ and $3 \mathrm{~h}$ ) shows that the major difference in morphology of the two groups of nanowires is the length distribution. The first group has the length range from $10 \mathrm{~nm}$ to $600 \mathrm{~nm}$ and the second group from $100 \mathrm{~nm}$ to $400 \mathrm{~nm}$. Magnetization measurements did not show a noticeable difference in coercivity (both are above $10 \mathrm{kOe}$ ). However one can find an obvious difference in the "squareness" ( 0.66 and 0.78 respectively, as shown in Fig. $3 \mathrm{c}$ and $3 \mathrm{~d})$. This difference has not been trivial in view of the energy product. In fact, the squareness of the demagnetization curves has the same important effect as the remanent magnetization and coercivity on the energy product (shown as the shadowed squares in Fig. $3 \mathrm{c}$ and $3 \mathrm{~d}$ ). It is striking to see that the small difference in the squareness has led to about a $25 \%$ difference in energy product of the two groups of samples. It is understood that in nanostructured ferromagnet systems, morphology uniformity plays an important role in magnetic reversal process. A narrowly distributed shape and nanowire length with diameters of appropriate dimension can lead to a coherent magnetization reversal process that gives rise to enhanced coercivity and squareness of the loops ${ }^{23}$.

\section{Conclusion}

In summary, record high room-temperature coercivity has been developed in nanocrystalline Co metal material with high aspect ratio, synthesized through a carefully controlled chemical solvother- 
a

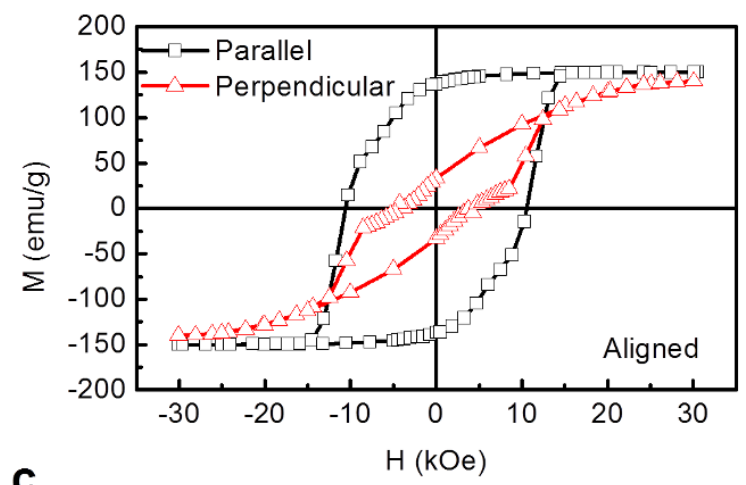

C

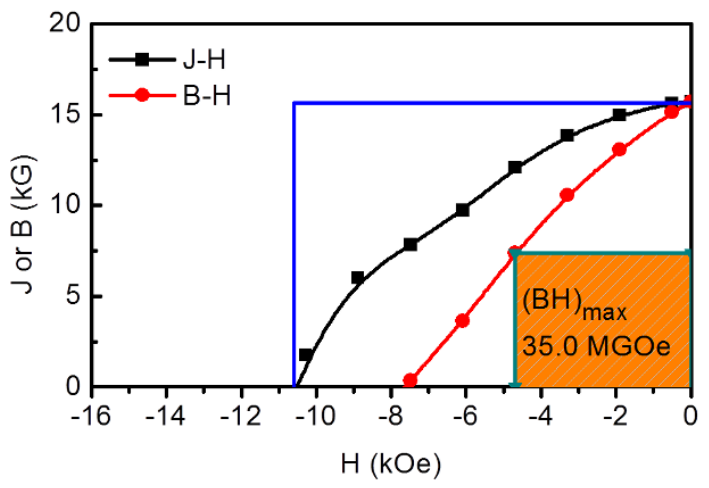

e

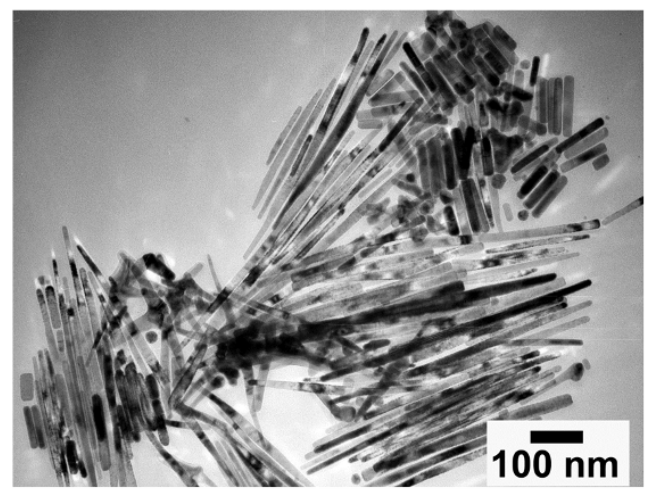

g

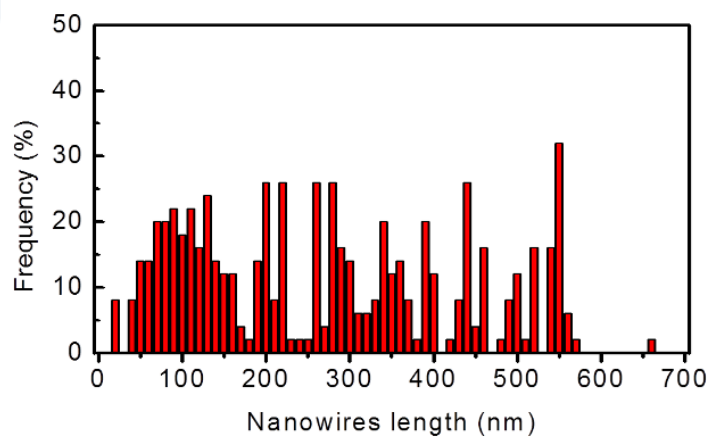

b

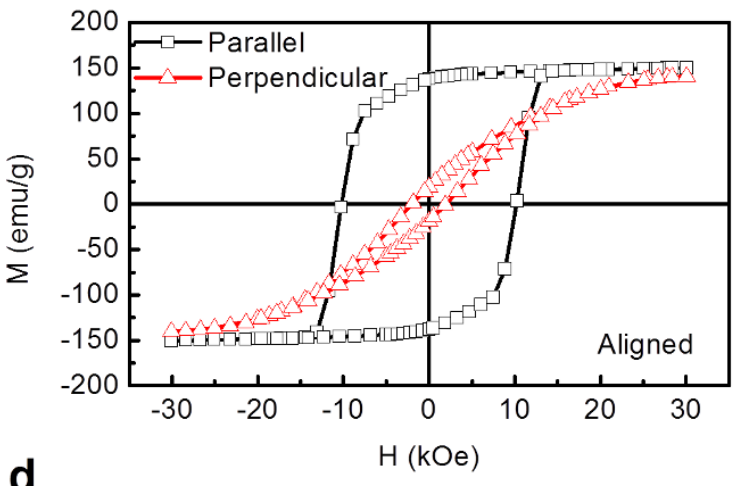

d

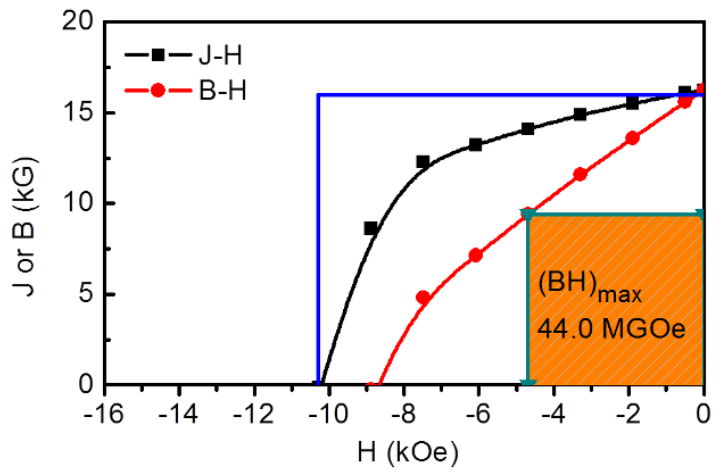

f

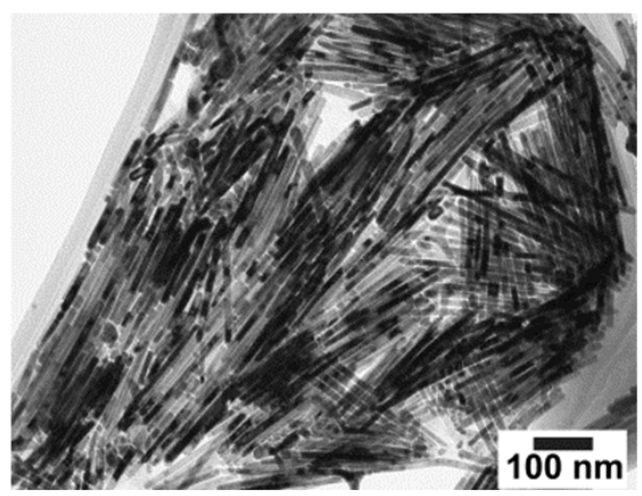

h

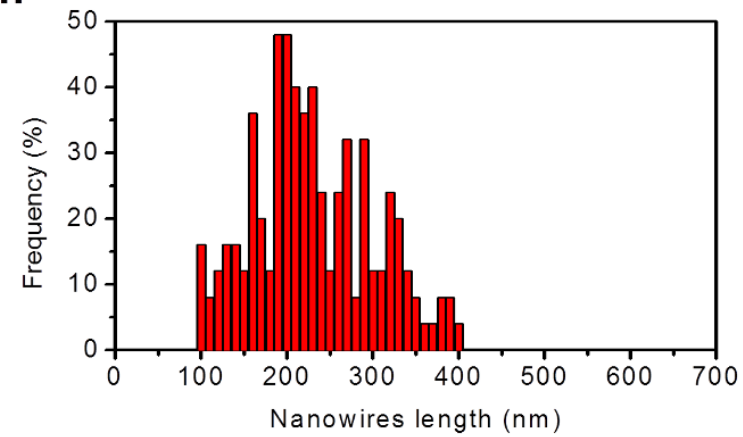

Figure 3 Magnetic hysteresis and morphology. (a), Magnetization loops of an aligned Co nanowire sample in epoxy (along parallel and perpendicular direction) at $300 \mathrm{~K}$. (b), Magnetization loops of aligned Co nanowires in epoxy (along parallel and perpendicular direction) at $300 \mathrm{~K}$. (c-d), Second quadrant B-H curves for the aligned Co nanowires in epoxy (along the easy axis) at $300 \mathrm{~K}$ of sample (a) and (b) respectively. (e-f), TEM images of the Co nanowires corresponding to samples (a) and (b) respectively. ( $\mathrm{g}-\mathrm{h}$ ), The histograms of the Co nanowires showing their length distributions of the samples shown in (e) and (f) respectively obtained using statistical analysis of $\sim 600$ nanowires each.

mal process, which resulted in high energy product in the material. Shape anisotropy provides the basis of the enhanced coercivity, and the orientation and uniformity of the single-domain nanocrystals are essential for achieving high magnetic energy density. Present experi- ments show that Co nanowires with diameters of $15 \mathrm{~nm}$ and an average length of $200 \mathrm{~nm}$ have a record high coercivity of $10.3 \mathrm{kOe}$ at room temperature, leading to an energy product of 44 MGOe. Ferromagnetic nanowires of this type are ideal building 
blocks for future bonded, consolidated and thin film magnets with high energy density and high thermal stability.

\section{Methods}

$\mathrm{CoCl}_{2} \cdot 6 \mathrm{H}_{2} \mathrm{O}$ (Alfa Aesar, 99.9\%), $\mathrm{RuCl}_{3}$ (Aldrich, $45-55 \% \mathrm{Ru}$ content), $\mathrm{NaOH}$ (Sigma Aldrich, $\geq 97 \%$ ), 1, 2- butanediol (Fluka, $\geq 98 \%$ ), Hexadecylamine (Aldrich, $98 \%$ ), methanol (VWR, Normapur) and lauric acid $\left(\mathrm{C}_{11} \mathrm{H}_{23} \mathrm{COO}\right)$ (Aldrich, 98\%) were used without any further purification.

Preparation of cobalt (II) laurate. The cobalt (II) laurate, $\mathrm{Co}\left(\mathrm{C}_{11} \mathrm{H}_{23} \mathrm{COO}\right)_{2}$, was prepared following a procedure adapted from a previously published synthesis for the cobalt (II) laurate salt ${ }^{15,24}$. Lauric acid $(44.0 \mathrm{mmol})$ and $\mathrm{NaOH}(42.0 \mathrm{mmol})$ was added to $40 \mathrm{ml}$ DI water while being mixed using a mechanical stirrer. The resulted mixture was heated to $60^{\circ} \mathrm{C}$ until a clear solution was obtained. At $60^{\circ} \mathrm{C}, 10 \mathrm{~mL}$ of an aqueous solution of $2 \mathrm{M} \mathrm{CoCl}_{2} \cdot 6 \mathrm{H}_{2} \mathrm{O}(99.9 \%)(20.0 \mathrm{mmol})$ was added drop wise to the solution with vigorous mechanical stirring. Afterwards, a purple precipitate formed, and the mixture was stirred and kept at $60^{\circ} \mathrm{C}$ for $30 \mathrm{~min}$ after the $\mathrm{CoCl}_{2} \cdot 6 \mathrm{H}_{2} \mathrm{O}$ addition. The precipitate was recovered by centrifugation ( $6000 \mathrm{rpm}$ for $15 \mathrm{~min}$ per wash), one time with $45 \mathrm{~mL}$ DI water and three times with $45 \mathrm{~mL}$ of methanol then dried in an air oven at $60^{\circ} \mathrm{C}$ followed by drying under vacuum.

Cobalt nanowires synthesis. In a typical synthesis, cobalt (II) laurate (2.07 g, $4.5 \mathrm{mmol}), \mathrm{RuCl}_{3}(0.0037 \mathrm{~g}, 0.018 \mathrm{mmol})$, Hexadecylamine (0.5810 g, $\left.2.4 \mathrm{mmol}\right)$ and $60 \mathrm{~mL}$ of 1,2 Butanediol were introduced inside a teflon enclosure $(100 \mathrm{~mL})$ with the $\mathrm{Ru} / \mathrm{Co}$ molar ratio fixed at $0.4 \%$. The teflon enclosure was purged with forming gas ( $\mathrm{Ar} 93 \%+\mathrm{H}_{2} 7 \%$ ) for $5 \mathrm{~min}$ then sealed. Afterwards, the enclosure was placed in a heated, ultrasonic water bath adjusted to $80^{\circ} \mathrm{C}$. The contents within the enclosure were then mixed for $60 \mathrm{~min}$ using the ultrasonic function of the water bath. The temperature of the water bath was maintained at $80^{\circ} \mathrm{C}$ throughout the mixing process. Next, the teflon enclosure was removed from the water bath and fitted within an autoclave reactor. The autoclave reactor was transferred to a furnace and heated from room temperature to $250^{\circ} \mathrm{C}$ at a rate of $8^{\circ} \mathrm{C}$ per minute then maintained at $250^{\circ} \mathrm{C}$ for $75 \mathrm{~min}$. After cooling to room temperature, the black powder consisting of cobalt nanowires was separated from reaction fluid by centrifugation at $6000 \mathrm{rpm}$ for $15 \mathrm{~min}$. The powder was collected then redispersed in $30 \mathrm{ml}$ toluene using an ultrasonic bath. The sample was centrifuged once again at $6000 \mathrm{rpm}$ for $15 \mathrm{~min}$ and the toluene discarded. This purification step was repeated two more times. After the purification, cobalt nanowires were dried in a vacuum oven at $50^{\circ} \mathrm{C}$ then stored within a glove box with Ar atmosphere.

Characterizations. The transmission electron microscopy (TEM) images were recorded on a JEOL 1200 EX electron microscope at an accelerating voltage of $120 \mathrm{kV}$. The nanowires samples were prepared evaporating the toluene dispersion on carbon-coated copper grids. High resolution TEM images were obtained with a Hitachi H-9500 high-resolution TEM with an accelerated voltage of $300 \mathrm{kV}$. Lacey carbon grids were used for HRTEM investigation. Electron holography image of single-cobalt nanowire was recorded digitally at an accelerating voltage of $200 \mathrm{kV}$ in a JEOL JEM-2100F-LM field emission gun TEM equipped with JEOL biprism $(0.6 \mu \mathrm{m}$ diameter, $180^{\circ}$ rotation), in a remanent field about 4 Oe. The reconstructed phase image of specimen provides a visual picture of magnetic flux in form of contours.

Powder X-ray diffraction (XRD) spectra were recorded on a Rigaku Ultima IV diffractometer with a $\mathrm{Cu} \mathrm{K}-\alpha$ wavelength X-ray source. Magnetic measurements of the metallic samples were performed using a Quantum Design MPMS magnetometer (SQUID). Randomly oriented Co nanowire samples for magnetic characterization were prepared by dispersing then curing in a rapid-set epoxy resin. Aligned Co nanowires were prepared by sonicating the toluene dispersion for $5 \mathrm{~min}$ then adding the epoxy into the Co nanowires/toluene dispersion and again sonicating for $2 \mathrm{~min}$. This composite was then poured into a mold and allowed to cure under the external magnetic field of $2.0 \mathrm{~T}$ in an electromagnet.

In order to determine the actual magnetization of the as synthesized Co nanowires before hardening into epoxy, inductively coupled plasma mass spectrometry (ICPMS) was used to quantitatively determine the mass of the Co metal in the nanowire samples. The Co nanowires were initially washed 3 times with toluene and dried in glove box to prevent oxidation. The dried samples were weighed in a vial then digested with $1 \mathrm{ml}$ of concentrated $\mathrm{HNO}_{3}$ at room temperature. After 1 hour, the solution was diluted down with $1 \% \mathrm{HNO}_{3}$ to the appropriate concentration for the ICP-MS analysis. The results indicate that Co nanowires contain (by weight) $85.4 \%$ of Co which was used to calibrate the magnetization values of the nanowires.

1. Buschow, K. Intermetallic compounds of rare-earth and $3 \mathrm{~d}$ transition metals. Reports on Progress in Physics, 1179. Rep. Prog. Phys. 40, 1179-1256 (1977).

2. Gutfleisch, O. et al. Magnetic materials and devices for the 21 st century: stronger, lighter, and more energy efficient. Adv. Mater. 23, 821-42 (2011).

3. Burkert, T., Nordström, L., Eriksson, O. \& Heinonen, O. Giant Magnetic Anisotropy in Tetragonal FeCo Alloys. Phys. Rev. Lett. 93, 027203 (2004).
4. Oliver, D. A. \& Shedden, J. W. Cooling of permanant magnetic alloy in a constant magnetic field. Nature 3587, 209 (1938).

5. Sun, L., Hao, Y., Chien, C.-L. \& Searson, P. C. Tuning the properties of magnetic nanowires. IBM J. RES. \& DEV. 49, 79-102 (2005).

6. Poudyal, N. et al. Synthesis of FePt nanorods and nanowires by a facile method. Nanotechnology 19, 355601 (2008).

7. Hao, R. et al. Synthesis, Functionalization, and Biomedical Applications of Multifunctional Magnetic Nanoparticles. Adv. Mater. 22, 2729-2742 (2010).

8. Zhang, Y. et al. Controlled synthesis and magnetic properties of hard magnetic $\mathrm{Co}_{\mathrm{x}} \mathrm{C}(\mathrm{x}=2,3)$ nanocrystals. J. Magn. Magn. Mater. 323, 1495-1500 (2011).

9. Gandha, K., Poudyal, N., Zhang, Q. \& Liu, J. P. Effect of $\mathrm{RuCl}_{3}$ on Morphology and Magnetic Properties of CoNi Nanowires. IEEE Trans. Magn. 49, 3273-3276 (2013).

10. Sellmyer, D. J., Zheng, M. \& Skomski, R. Magnetism of Fe, Co and Ni nanowires in self-assembled arrays. J. Phys. Condens. Matter 13, R433-R460 (2001).

11. Su, H. L. et al. Geometry dependence of the annealing effect on the magnetic properties of $\mathrm{Fe}_{48} \mathrm{Co}_{52}$ nanowire arrays. Nanotechnology 16, 429-432 (2005).

12. Cattaneo, L. et al. Electrodeposition of hexagonal Co nanowires with large magnetocrystalline anisotropy. Electrochim. Acta 85, 57-65 (2012).

13. Maurer, T. et al. Magnetic nanowires as permanent magnet materials. Appl. Phys. Lett. 91, 172501 (2007).

14. Dumestre, F. et al. Shape control of thermodynamically stable cobalt nanorods through organometallic chemistry. Angew. Chem. Int. Ed. 41, 4286-9 (2002).

15. Soumare, Y. et al. Kinetically Controlled Synthesis of Hexagonally Close-Packed Cobalt Nanorods with High Magnetic Coercivity. Adv. Funct. Mater. 19, 1971-1977 (2009).

16. Soulantica, K. et al. Magnetism of single-crystalline Co nanorods. Appl. Phys. Lett. 95, 152504 (2009).

17. Fang, W. et al. Optimization of the magnetic properties of aligned Co nanowires/ polymer composites for the fabrication of permanent magnets. J. Nanopart. Res. 16, 2265 (2014).

18. Cullity, B. D. \& Graham, C. D. Introduction to Magnetic Materials $2^{\text {nd }}$ edition (John Wiley \& Sons, Inc., 2009).

19. Skomski, R. \& Coey, J. M. D. Permanent Magnetism (Taylor \& Francis Group, 1999).

20. Stoner, E. C. \& Wohlfarth, E. P. A mechanism of magnetic hysteresis in heterogeneous alloys. Philos. Trans. R. Soc. London A240, 599-642 (1948).

21. Ait-Atmane, K. et al. High temperature structural and magnetic properties of cobalt nanorods. J. Solid State Chem. 197, 297-303 (2013).

22. Panagiotopoulos, I. et al. Packing fraction dependence of the coercivity and the energy product in nanowire based permanent magnets. J. Appl. Phys. 114, 143902 (2013).

23. Skomski, R., Liu, Y., Shield, J. E., Hadjipanayis, G. C. \& Sellmyer, D. J. Permanent magnetism of dense-packed nanostrucutres. J. Appl. Phys. 107, 09A739 (2010).

24. Kulkarni, S., Alurkar, M. \& Kumar, A. Polymer support with Schiff base functional group with cobaltous palmitate as oxidation catalyst for cyclohexane. Appl. Catal. A 142, 243 (1996).

\section{Acknowledgments}

This work has been supported by the DoD/ARO under grant W911NF-11-1-0507, and the Center for Nanostructured Materials and Characterization Center for Materials and Biology at the University of Texas at Arlington. The authors thank Drs. Jason Zhang, Max $\mathrm{Hu}$ and Weixing Xia for their experimental support and fruitful discussions in the chemical sythesis, ICP measurements and holography observation.

\section{Author contributions}

K.G., K.E. and N.P. carried the experiments and data processing, J.P.L. supervised the project, X.L. and J.P.L. wrote the manuscript.

\section{Additional information}

Competing financial interests: The authors declare no competing financial interests. How to cite this article: Gandha, K., Elkins, K., Poudyal, N., Liu, X.B. \& Liu, J.P. High Energy Product Developed from Cobalt Nanowires. Sci. Rep. 4, 5345; DOI:10.1038/ srep05345 (2014) 